\title{
Adaptive B-Spline Scheme for Solving an Inverse Scattering Problem
}

\author{
Alexandre Baussard ${ }^{1} \ddagger$, Eric L. Miller ${ }^{2}$, Denis Prémel $^{1} \S$ \\ ${ }^{1}$ SATIE (CNRS UMR-8029) - ENS de Cachan, 61 avenue du Président Wilson, \\ 94235 Cachan Cedex, France. \\ ${ }^{2}$ CenSSIS - Northeastern University, 315 Stearns Hall, 360 Huntington Ave., Boston \\ MA 02115-5000, USA.
}

\begin{abstract}
In this paper, we present an approach to the 2D inverse scattering problem in which the unknown object is well approximated using a small number of adaptively chosen B-spline basis functions. Rather than determining a large collection of pixel values as is commonly done, we estimate directly a much smaller knot sequence associated with a B-spline representation of the object. An iterative scheme is proposed in which, at each stage, we seek to improve our estimate of the object through the insertion and deletion of knots. A specific knot insertion process, based on curvature information, and a specific knot deletion method, based on data fitting, are proposed. Given a collection of knots, a non-linear, conjugate-gradient method is employed to determine an estimate of the object. By controlling the degrees of freedom in this manner we are able to forgo an explicit regularization scheme. Finally, using both computer simulated as well as experimental microwave laboratory-controlled data, we demonstrate the ability of our approach to improve upon that obtained from a more standard, pixel-based inverse scheme.
\end{abstract}

Submitted to: Inverse Problems

$\ddagger$ is now with L2S (CNRS/SUPELEC/UPS), 3 rue Joliot Curie, 91192 Gif-sur-Yvette France, Email: baussard@lss.supelec.fr $\S$ is now with CEA Saclay, DRT/DECS/SISC/LCME, 91191 Gif-sur-Yvette, France. 


\section{Introduction}

Various applications in fields such as nondestructive evaluation, medical imaging, and geophysical prospecting are concerned with the determination of the spatial variation of some physical properties of an unknown and compactly supported object from measurements of the scattered response of a known electromagnetic or acoustic excitation. Several algorithms, such as those in $[8,11,17]$, have already been developed to solve the corresponding non-linear and ill-posed inverse problem. These approaches require the solution of a large-scale, non-linear optimization problem the size of which corresponds to the number of pixels or voxels used to discretize the search area. These algorithms generate values for every pixel, including those not associated with the object itself thereby accentuating the ill-posed nature of the problem.

In this paper, to reduce the complexity (i.e., the dimensionality) of the problem, an adaptive B-spline approach is proposed and applied to a two-dimensional scalar inverse scattering problem. B-splines have already shown their usefulness in interpolation, approximation and they have been widely used in computer-aided geometric design (CAGD) [1, 3, 6, 9]. They have successfully been also used in a one-dimensional, rough-surface inversion [13] and, in previous work, for solving a one-dimensional inverse scattering problem for a diffusion coefficient [15] and for a two-dimensional shape-based approach for the localization of buried objects [14]. In this contribution, the use of tensors products of B-splines, defined in terms of their knot distributions, provides a potentially useful means of parameterizing the unknown object.

The primary objective of the proposed method is to construct adaptively an efficient an accurate and low-order parameterization of the object using a small number of knots. Roughly speaking, such a distribution is characterized by few knots in flat regions of the object and a higher density of knots in regions of more "activity" such as edges. Then, the size of the problem one has to solve would only depend on the number of knots in our representation, a quantity many times smaller than the corresponding number of pixels. Several knot insertion and deletion procedures have been developed for CAGD, interpolation or approximation. In this work, a knot insertion procedure based on curvature information and a deletion procedure based on data fitting are proposed. These knot refinement processes are embedded in an overall inversion strategy comprised of a sequence of very low-order, non-linear optimization steps. Specifically, given a collection of knots as determined by our refinement process, the optimization step is solved using a conjugate-gradient method [7, 12].

Moreover, as exemplified using both synthetic and real data, our reduced order and adaptive representation can improve the quality of the recovered object for the class of problem of our interest. In fact, using low order B-spline parameterization of the unknown imposes a degree of smoothness to the reconstructed image which removes the need for explicit regularization using e.g., a Tikhonov-type functional.

The paper is organized as follows. In section 2 , the formulation of the problem is presented. Section 3 presents the pixel-based, non-linear inversion procedure. Section 
4 introduces the B-spline formulation. Section 5 and 6 describe the knot insertion and deletion processes, respectively. Section 7 shows some results obtained using simulated data and in section 8 the proposed algorithm is tested against laboratory-controlled data, both in the microwave domain. Finally, section 9 gives a brief overview of the proposed approach and some concluding remarks.

\section{Formulation of the problem}

The geometry of the problem is presented in Figure 1. A two-dimensional object of arbitrary cross-section is illuminated by a given incident field and the scattered field is measured on receivers all around the object along a contour $\Gamma$. A search domain $\Omega$ containing the object is considered. The embedding medium $\Omega_{b}$ is assumed to be of infinite extent and homogeneous, with a given permittivity $\varepsilon_{b}=\varepsilon_{0} \varepsilon_{b r}$, and permeability $\mu=\mu_{0}$ ( $\varepsilon_{0}$ and $\mu_{0}$ being the permittivity and permeability of the vacuum, respectively). The object is assumed to be an inhomogeneous non-magnetic cylinder with complexvalued permittivity distribution $\varepsilon(\mathbf{r})=\varepsilon_{0} \varepsilon_{r}(\mathbf{r})$.

The sources that generate the electromagnetic excitation, Transverse Magnetic (TM) polarized, are assumed to be lines parallel to the $z$-axis, located at $\left(\mathbf{r}_{l}\right)_{1 \leq l \leq L}$. With an assumed $\exp (-i \omega t)$ time dependence, the time-harmonic incident electric field created by the $l^{\text {th }}$ source is given by:

$$
e_{l}^{i n c}(\mathbf{r})=P \frac{\omega \mu_{0}}{4} H_{0}^{(1)}\left(k_{b}\left|\mathbf{r}-\mathbf{r}_{l}\right|\right),
$$

where $P$ is the strength of the electric source, $\omega$ is the angular frequency, $H_{0}^{(1)}$ is the first-kind, zero-order Hankel function and $k_{b}$ is the wavenumber in the surrounding medium.

For the inverse scattering problem we assume that the unknown object is successively illuminated by $L$ electromagnetic excitations and for each incident field the scattered field is available at $M$ positions. For each excitation, the forward problem is described by two coupled contrast-source integral equations, the observation equation:

$$
e_{l}^{d}(\mathbf{r} \in \Gamma)=k_{b}^{2} \int_{\Omega} \chi\left(\mathbf{r}^{\prime}\right) e_{l}\left(\mathbf{r}^{\prime}\right) G\left(\mathbf{r}, \mathbf{r}^{\prime}\right) \mathrm{d} \mathbf{r}^{\prime}
$$

and the coupling equation:

$$
e_{l}(\mathbf{r} \in \Omega)=e_{l}^{i n c}(\mathbf{r})+k_{b}^{2} \int_{\Omega} \chi\left(\mathbf{r}^{\prime}\right) e_{l}\left(\mathbf{r}^{\prime}\right) G\left(\mathbf{r}, \mathbf{r}^{\prime}\right) \mathrm{d} \mathbf{r}^{\prime},
$$

where $\chi(\mathbf{r})=\varepsilon_{r}(\mathbf{r})-\varepsilon_{b r}$ denotes the contrast function, $G\left(\mathbf{r}, \mathbf{r}^{\prime}\right)$ is the two-dimensional homogeneous free-space Green function, and $k_{b}$ is the wavenumber.

The coupled integral equations (2) and (3) are discretized by using the method of moments (of the segment-segment type) [10]. The discretization is done by subdividing the search domain into a regular mesh, $\left[N_{x} \times N_{y}\right]$ sized. Then, for each source $l$, the two coupled equations can be written as the following matrix system:

$$
\mathbf{e}_{l}^{i n c}=\mathbf{e}_{l}-\mathbf{G}_{\Omega} D(\mathbf{c}) \mathbf{e}_{l},
$$




$$
\mathbf{e}_{l}^{d}=\mathbf{G}_{\Gamma} D\left(\mathbf{e}_{l}\right) \mathbf{c},
$$

where $\mathbf{e}_{l}$ is the total field vector of dimension $N\left(=N_{x} \times N_{y}\right), \mathbf{e}_{l}^{i n c}$ is the incident field vector of dimension $N, \mathbf{e}_{l}^{d}$ is the scattered field vector of dimension $M$, $\mathbf{c}$ is the contrast vector of dimension $N$ and $\mathbf{G}_{\Omega}$ and $\mathbf{G}_{\Gamma}$ are the $[N \times N]$ and $[M \times N]$ matrices made of properly integrated Green's functions. $D(\mathbf{t})$ denotes a diagonal matrix whose entries are the elements of a vector $\mathbf{t}$.

These two coupled equations can be expressed as the following non-linear equation in $\mathbf{c}$ :

$$
\mathbf{e}_{l}^{d}=\mathbf{A}_{l}(\mathbf{c})+\mathbf{b},
$$

where $\mathbf{A}_{l}(\mathbf{c})=\mathbf{G}_{\Gamma} D\left[\left(\mathbf{I}_{d}-\mathbf{G}_{\Omega} D(\mathbf{c})\right)^{-1} \mathbf{e}_{l}^{i n c}\right] \mathbf{c}$ and where $\mathbf{b}$ stands for the noise, assumed to be additive. $I_{d}$ is the $[N \times N]$ identity matrix. Moreover, in what follows, we define $\Theta(\mathbf{c})=\left(\mathbf{I}_{d}-\mathbf{G}_{\Omega} D(\mathbf{c})\right)^{-1}$.

The problem is now to retrieve the contrast profile $\mathbf{c}$ from the given scattered fields $\mathbf{e}_{l}^{d}$.

\section{The non-linear inversion procedure}

In order to solve the corresponding non-linear inverse scattering problem, a conjugategradient method $[7,12]$ is applied. Considering (6), one introduces the cost function:

$$
J(\mathbf{c})=\sum_{l=1}^{L}\left\|\rho_{l}(\mathbf{c})\right\|^{2},
$$

where $\rho_{l}(\mathbf{c})=\mathbf{e}_{l}^{d}-\mathbf{A}_{l}(\mathbf{c})$ is the residual vector. Eq. (7) represents the error when matching the scattered field data.

Because the object to be estimated is complex-valued (the real part represents the permittivity and the imaginary part is associated with the conductivity), following [7] the contrast function reads as:

$$
\chi=\xi+i \eta-\varepsilon_{b r},
$$

where $\xi$ and $\eta$ are two real-valued auxiliary parameters. Then, the optimization problem defined in (7) is transformed into the minimization of the function $J$ depending on the two auxiliary vector parameters $\mathbf{c}_{\xi}$ and $\mathbf{c}_{\eta}$ :

$$
J\left(\mathbf{c}_{\xi}, \mathbf{c}_{\eta}\right)=\sum_{l=1}^{L}\left\|\rho_{l}\left(\mathbf{c}_{\xi}, \mathbf{c}_{\eta}\right)\right\|^{2} .
$$

In order to find a minimizer of $J\left(\mathbf{c}_{\xi}, \mathbf{c}_{\eta}\right)$, two sequences $\left\{\mathbf{c}_{\xi, n}\right\}$ and $\left\{\mathbf{c}_{\eta, n}\right\}$ are constructed using the following iterative relations:

$$
\begin{gathered}
\mathbf{c}_{\xi, n}=\mathbf{c}_{\xi, n-1}+\alpha_{\xi, n} \mathbf{d}_{\xi, n}, \\
\mathbf{c}_{\eta, n}=\mathbf{c}_{\eta, n-1}+\alpha_{\eta, n} \mathbf{d}_{\eta, n},
\end{gathered}
$$


where all quantities are real-valued. The coefficients $\alpha_{\xi, n}$ and $\alpha_{\eta, n}$ are determined at each iteration using the derivative Brent method [16]. The (vector) search directions $\mathbf{d}_{\xi, n}$ and $\mathbf{d}_{\eta, n}$ are the standard Polak-Ribière conjugate gradient directions:

$$
\begin{aligned}
\mathbf{d}_{\xi, n}=\mathbf{g}_{\xi, n}+\gamma_{\xi, n} \mathbf{d}_{\xi, n-1} \quad \text { with } \quad \gamma_{\xi, n}=\frac{\left\langle\mathbf{g}_{\xi, n} \mid \mathbf{g}_{\xi, n}-\mathbf{g}_{\xi, n-1}\right\rangle_{\Omega}}{\left\|\mathbf{g}_{\xi, n-1}\right\|_{\Omega}^{2}} \\
\mathbf{d}_{\eta, n}=\mathbf{g}_{\eta, n}+\gamma_{\eta, n} \mathbf{d}_{\eta, n-1} \quad \text { with } \quad \gamma_{\eta, n}=\frac{\left\langle\mathbf{g}_{\eta, n} \mid \mathbf{g}_{\eta, n}-\mathbf{g}_{\eta, n-1}\right\rangle_{\Omega}}{\left\|\mathbf{g}_{\eta, n-1}\right\|_{\Omega}^{2}},
\end{aligned}
$$

where $<. \mid .>_{D}$ represent the inner product defined on $L^{2}(D)$ and $\mathbf{g}_{\xi, n}$ and $\mathbf{g}_{\eta, n}$ are the gradients of the cost functional $J\left(\mathbf{c}_{\xi}, \mathbf{c}_{\eta}\right)$ with respect to $\mathbf{c}_{\xi, n}$ and $\mathbf{c}_{\eta, n}$, respectively. These gradients are given by:

$$
\begin{aligned}
& \mathbf{g}_{\xi, n}=-2 \sum_{l=1}^{L} \operatorname{Re}\left[D\left(\overline{\Theta(\mathbf{c}) \mathbf{e}_{l}^{i n c}}\right)\left[I_{d}+D(\mathbf{c}) \Theta(\mathbf{c}) \mathbf{G}_{\Omega}\right]^{\dagger} \mathbf{G}_{\Gamma}^{\dagger} \rho_{l}\left(\mathbf{c}_{\xi}, \mathbf{c}_{\eta}\right)\right] \\
& \mathbf{g}_{\eta, n}=-2 \sum_{l=1}^{L} \operatorname{Im}\left[D\left(\overline{\Theta(\mathbf{c}) \mathbf{e}_{l}^{i n c}}\right)\left[I_{d}+D(\mathbf{c}) \Theta(\mathbf{c}) \mathbf{G}_{\Omega}\right]^{\dagger} \mathbf{G}_{\Gamma}^{\dagger} \rho_{l}\left(\mathbf{c}_{\xi}, \mathbf{c}_{\eta}\right)\right]
\end{aligned}
$$

where $\overline{\mathrm{T}}$ denotes the complex conjugate of $\mathrm{T}$, while $\mathrm{T}^{\dagger}$ denotes the adjoint operator of T.

\section{Adaptive B-spline approach}

\subsection{Bivariate spline overview}

The most direct extension of the univariate splines to the bivariate case is via a tensor product. In accord with $[1,6]$, let us consider the strictly increasing sequences:

$$
\begin{aligned}
& a=\lambda_{0}<\lambda_{1}<\ldots<\lambda_{g+1}=b, \\
& c=\mu_{0}<\mu_{1}<\ldots<\mu_{h+1}=d .
\end{aligned}
$$

The function $s(x, y)$ is a bivariate spline on $[a, b] \times[c, d]$ of degree $k$ (order $k+1$ ) in $x$ and $l$ (order $l+1$ ) in $y$, with knots $\lambda_{i}, i=0, \ldots, g+1$, in the $x$-direction and $\mu_{i}$, $i=0, \ldots, h+1$, in the $y$-direction, if the following properties are satisfied:

- On each subrectangle $\left[\lambda_{i}, \lambda_{i+1}\right] \times\left[\mu_{j}, \mu_{j+1}\right], s(x, y)$ is given by a polynomial of degree $k$ in $x$ and $l$ in $y$ at least:

$$
s_{\left[\lambda_{i}, \lambda_{i+1}\right] \times\left[\mu_{j}, \mu_{j+1}\right]} \in \mathbb{P}_{k} \otimes \mathbb{P}_{l},
$$

with $i=0, \ldots, g ; j=0, \ldots, h$.

- The function $s(x, y)$ and its partial derivatives are all continuous on $[a, b] \times[c, d]$ :

$$
\frac{\partial^{i+j} s(x, y)}{\partial x^{i} \partial y^{j}} \in \mathbb{C}([a, b] \times[c, d]),
$$

with $i=0, \ldots, k-1 ; j=0, \ldots, l-1$. 
Given the above set of knots, the collection of functions which satisfies the specified properties forms a vector space, denoted by $\eta_{k, l}\left(\lambda_{0}, . ., \lambda_{g+1} ; \mu_{0}, . ., \mu_{h+1}\right)$, of dimension $(g+k+1)(h+l+1)$. From the univariate case (see [6]), the following boundary knots are introduced:

$$
\begin{aligned}
& \lambda_{-k}<\lambda_{-k+1}<\ldots<\lambda_{0}=a, \\
& b=\lambda_{g+1}<\lambda_{g+2}<\ldots<\lambda_{g+k+1}, \\
& \mu_{-l}<\mu_{-l+1}<\ldots<\mu_{0}=c, \\
& d=\mu_{h+1}<\mu_{h+2}<\ldots<\mu_{h+l+1},
\end{aligned}
$$

so that any tensor product spline $s(x, y) \in \eta_{k, l}\left(\lambda_{0}, . ., \lambda_{g+1} ; \mu_{0}, . ., \mu_{h+1}\right)$ has a unique representation:

$$
s(x, y)=\sum_{i=-k}^{g} \sum_{j=-l}^{h} a_{i, j} N_{i, k+1}(x) M_{j, l+1}(y),
$$

where $N_{i, k+1}(x)$ and $M_{j, l+1}(y)$ are the (normalized) B-splines and $a_{i, j}$ are the B-spline coefficients of $s(x, y)$.

One recalls that the B-spline $B_{i, k+1}(x)$ of degree $k$ with knots $t_{i}, . ., t_{i+k+1}$ is defined as:

$$
B_{i, k+1}(x)=\left(t_{i+k+1}-t_{i}\right) \sum_{j=0}^{k+1} \frac{\left(t_{i+j}-x\right)_{+}^{k}}{\prod_{l=0, l \neq j}^{k+1}\left(t_{i+j}-t_{i+l}\right)},
$$

where

$$
(t-x)_{+}^{k}= \begin{cases}(t-x)^{k} & \text { if } t \geq x \\ 0 & \text { otherwise. }\end{cases}
$$

From the univariate case, the tensor product B-spline $N_{i, k+1}(x) M_{j, l+1}(y)$ has the following properties:

$$
\begin{aligned}
& N_{i, k+1}(x) M_{j, l+1}(y)\left\{\begin{array}{l}
\geq 0 \text { for } x, y \in[a, b] \times[c, d] \\
=0 \text { for } x, y \notin\left[\lambda_{i}, \lambda_{i+1}\right] \times\left[\mu_{j}, \mu_{j+1}\right]
\end{array},\right. \\
& \sum_{i=-k}^{g} \sum_{j=-l}^{h} N_{i, k+1}(x) M_{j, l+1}(y)=1 \text { for } x, y \in[a, b] \times[c, d] .
\end{aligned}
$$

\subsection{Adaptive inversion}

The proposed approach is rather simple in principle since it consists in reducing the complexity of the inverse problem by approximating the object using splines. The contrast is then defined as:

$$
\mathbf{c}=\mathbf{B a}
$$

where $\mathbf{B}$ is the (sparse) matrix made of cubic B-splines and $\mathbf{a}$ is a vector of expansion coefficients.

Thus, the problem can be reformulated as:

$$
\mathbf{e}_{l}^{d}=\mathbf{A}_{l}(\mathbf{a})+\mathbf{b},
$$


where $\mathbf{A}_{l}(\mathbf{a})=\mathbf{G}_{\Gamma} D\left[\left(\mathbf{I}_{d}-\mathbf{G}_{\Omega} D(\mathbf{B a})\right)^{-1} \mathbf{e}_{l}^{i n c}\right] \mathbf{B a}$. The corresponding non-linear inversion is solved by considering the following cost functional:

$$
J(\mathbf{a})=\sum_{l=1}^{L}\left\|\rho_{l}(\mathbf{a})\right\|^{2},
$$

where $\rho_{l}(\mathbf{a})=\mathbf{e}_{l}^{d}-\mathbf{G}_{\Gamma} D\left[\left(\mathbf{I}_{d}-\mathbf{G}_{\Omega} D(\mathbf{B a})\right)^{-1} \mathbf{e}_{l}^{i n c}\right] \mathbf{B a}$.

As for the complex contrast function $\chi$, a can be split in two as:

$$
\mathbf{a}=\mathbf{a}_{\xi}+i \mathbf{a}_{\eta}-\varepsilon_{b r} .
$$

The initial inversion problem, i.e., the retrieval of the auxiliary vectors $\mathbf{c}_{\xi}$ and $\mathbf{c}_{\eta}$, both vectors being of dimension $N$, is transformed into the retrieval of the two auxiliary vectors $\mathbf{a}_{\xi}$ and $\mathbf{a}_{\eta}$ of dimension $N_{1}$ and $N_{2}$, respectively, depending on the number of knots, where $N_{1}<<N$ and $N_{2}<<N$. In what follows, to simplify the implementation of the algorithm and assuming either dielectric or metallic objects, only the real part or the imaginary part is considered. This prior consideration does not affect the general features of the analysis.

The problem is now to determine an appropriate knot distribution. On the one hand, one seeks a compact representation of the object to control complexity. On the other hand, one wishes to have sufficient flexibility in the representation of the object to capture its salient structure. This leads us to consider a multi-scale type of approach. Starting with a coarse collection of spline basis defined with relatively few knots, one obtains a coarse estimate of the object. Then, the spline functions are refined inside what it believed to be "interesting regions" (i.e. objects inside the search domain) in order to allow for improvements in the reconstruction. In the event that new or old knots are inefficient for the image representation, a method for removing them is also incorporated. The steps of the proposed global procedure are as follows:

S1 - Initialization steps:

S1.1 - Fix a collection of few knots, along the $x$-axis and $y$-axis, which define a coarse representation of the search domain without taking into account any $a$ priori information concerning the object locations (e.g. figure 6a).

S1.2 - Construct B and initialize a to zero.

S1.3 - Solve the corresponding non-linear inversion by minimizing (22).

S2 - Iterative procedure:

S2.1 - From the previous estimate of a, insert new knots in areas of interest. Such areas are identified by curvature information. This step is detailed in Section 5.

S2.2 - Delete the inefficient knots for the object representation (see section 6 for a detailed description).

S2.3 - Construct B corresponding to the new knot distribution.

S2.4 - Solve the corresponding non-linear inverse problem using the previous estimate of a as initial solution. 
As the first distribution of knots is a coarse representation, few iterations at the inversion step S1.3 are necessary. Moreover, for the other non-linear inversions (step S2.4), a maximum number of iterations is fixed as a parameter at the start of the algorithm. The global procedure is stopped when the difference between two successive criterion values is smaller than a fixed value (about $1.10^{-6}$ ), or when the maximum number of iterations for the step S2 (fixed at the beginning of the algorithm) is reached.

The key steps in this approach is to determine a useful knot insertion scheme (step S2.1) and deletion scheme (step S2.2). Both are detailed below.

\section{Knot insertion}

Different knot insertion schemes have already been developed for approximation, interpolation or CAGD, but these methods seem to be not really convenient for the problem. The goal of our knot insertion process (step S2.1) is to efficiently introduce new knots in "interesting areas". A method based on mean curvature information, which can be easily done by dealing with splines, is proposed.

\subsection{Curvature computation}

Let us briefly introduce, using [9], the mean curvature computation of a surface curve. The surface may be given by its parametric form as:

$$
\mathbf{x}=\left[\begin{array}{l}
x(u, v) \\
y(u, v) \\
z(u, v)
\end{array}\right] ; \mathbf{u}=\left[\begin{array}{l}
u \\
v
\end{array}\right] \in[\mathbf{a}, \mathbf{b}] \subset \mathbb{R}^{2},
$$

where the Cartesian coordinates $x, y, z$ of a surface point are function of parameters $u$ and $v$ and $[\mathbf{a}, \mathbf{b}]$ denotes a rectangle in the $(u, v)$ plane (see Figure 2). To avoid potential problems with undefined normal vectors, one assumes:

$$
\mathbf{x}_{u} \wedge \mathbf{x}_{v} \neq \mathbf{0} \text { for } \mathbf{u} \in[\mathbf{a}, \mathbf{b}] .
$$

where $\mathbf{x}_{u}=\frac{\mathrm{d} x}{\mathrm{~d} u}$ and $\mathbf{x}_{v}=\frac{\mathrm{d} x}{\mathrm{~d} v}$.

For a regular curve $\mathbf{x}[\mathbf{u}(t)]$ on the surface, the (squared) arc element is defined as:

$$
\mathrm{d} s^{2}=E \mathrm{~d} u^{2}+2 F \mathrm{~d} u \mathrm{~d} v+G \mathrm{~d} v^{2},
$$

where:

$$
\begin{aligned}
& E=E(u, v)=\mathbf{x}_{u} \cdot \mathbf{x}_{u}, \\
& F=F(u, v)=\mathbf{x}_{u} \cdot \mathbf{x}_{v}, \\
& G=G(u, v)=\mathbf{x}_{v} \cdot \mathbf{x}_{v} .
\end{aligned}
$$

Recall that the arc element $\mathrm{d} s$, being a geometric invariant of the curve through the point $\mathbf{x}$, does not depend on the particular parameterization chosen for the representation (24) of the surface.

To facilitate the description of local curve properties at a point $\mathbf{x}(t)$ on the curve, a local coordinate system (see Figure 3 ) is introduced. Let $\mathbf{x}_{u}$ and $\mathbf{x}_{v}$ be its axes, with 
origin $\mathbf{x}$. The partials $\mathbf{x}_{u}$ and $\mathbf{x}_{v}$ span the tangent plane to the surface at $\mathbf{x}$. Its normal $\mathbf{x}_{u} \wedge \mathbf{x}_{v}$ coincides with the normal to the surface at $\mathbf{x}$. The normalized normal $\mathbf{n}$ is defined as:

$$
\mathbf{n}=\frac{\mathbf{x}_{u} \wedge \mathbf{x}_{v}}{\left\|\mathbf{x}_{u} \wedge \mathbf{x}_{v}\right\|}
$$

Let now $\mathbf{u}(t)$ be a curve on the surface $\mathbf{x}(\mathbf{u})$. From curve theory and Meusnier's theorem (see [9] for more details), the normal curvature $\kappa$ of a surface curve at $\mathbf{x}$ in the direction $\mathbf{t}$, defined as $\frac{\mathrm{d} u}{\mathrm{~d} v}=\lambda$, is given by:

$$
\kappa(\lambda)=\frac{L+2 M \lambda+N \lambda^{2}}{E+2 F \lambda+G \lambda^{2}} .
$$

where $L=\mathbf{n} \cdot \mathbf{x}_{u u}, M=\mathbf{n} \cdot \mathbf{x}_{u v}$ and $N=\mathbf{n} \cdot \mathbf{x}_{v v}$.

In the general case, $\kappa(\lambda)$ is a rational quadratic function whose extreme values $\kappa_{1}$ and $\kappa_{2}$ (principal curvatures of the surface at $\mathbf{x}$ ) occur at the roots $\lambda_{1}$ and $\lambda_{2}$ (principal directions in the tangent plane) of:

$$
\operatorname{det}\left[\begin{array}{cc}
\kappa E-L & \kappa F-M \\
\kappa F-M & \kappa G-N
\end{array}\right]=0 .
$$

The mean curvature follows as:

$$
M_{c}=\frac{1}{2}\left(\kappa_{1}+\kappa_{2}\right) .
$$

\subsection{Knot insertion}

During the insertion, the added knots must provide a refined representation of the sought objects. The proposed steps are as follows:

S2.1.1 - From a current estimate of the (spline) object, compute the normalized mean curvature map.

S2.1.2 - Apply a threshold $T_{h}$, relative to a percentage of the maximum value of the curvature, on the map to find the "interesting areas" (objects inside the search domain).

S2.1.3 - Insert new knots between the old ones within these areas.

In the proposed insertion procedure, a threshold must be fixed. Large threshold values do not allow for the type of refinement sought in terms of best resolving the structure of the object. Small values of $T_{h}$ lead to the introduction of a large number of knots, not all of them being required to represent the true objects. While these "inefficient" knots will be removed during the deletion procedure (step S2.2) the practical desire of controlling the computation time at this step leads us to enforce enforces a minimum spacing between pairs of inserted knots. One can notice here that the location of the knots is referenced in terms of (center) numbered pixel (see the section on the numerical results). 


\section{Knot deletion}

The goal is to remove knots in a way that does the least damage to the accuracy of the reconstruction as measured by the cost function. That is, removal of knots tends to decrease the fidelity of the representation of the object thereby impacting the value of the cost function. Thus, one defines as "inefficient" knots those which do not significantly alter it.

To find the inefficient knots, a "weight" is computed for each knot of the distribution. Specifically, one removes a knot from the distribution and computes the cost, $J$, associated with this lower order representation of the object. The difficulty here is that, in principal, one must solve a non-linear optimization problem for each knot removed. To avoid the practical computational problems associated with such a strategy one notes that the removal of any one knot typically induces a "small" change in the object and hence we are motivated to solve linearized inverse problems at this stage of the algorithm in which the total field is held fixed at the value obtained with the full knot sequence. This 'linearization' does not directly influence the reconstruction in the sense that here one just looks for a value information on the weight of knots.

Formally, to compute the weight of each knot and to delete the inefficient ones, the following steps are proposed:

S2.2.1 - From the previous estimate of a and the knot distribution obtained after the insertion process (step $\mathbf{S 2 . 1}$ ), compute the total field $\mathbf{e}_{l}$ using (4).

S2.2.2 - Minimize the following (linearized) criterion:

$$
J^{\prime}(\mathbf{a})=\sum_{l=1}^{L}\left\|\rho_{l}^{\prime}(\mathbf{a})\right\|^{2},
$$

where $\rho_{l}^{\prime}(\mathbf{a})=\mathbf{e}_{l}^{d}-\mathbf{G}_{\Gamma} D\left(\mathbf{e}_{l}\right) \mathbf{B a}$. This gives the reference criterion value $J_{r e f}^{\prime}$.

S2.2.3 - Remove a knot from the distribution (except the extremum knots).

S2.2.4 - Minimize the criterion (32) and associate the obtained criterion value $J_{k n o t}^{\prime}$ to the removed knot.

S2.2.5 - Put back the knot and go to step S2.2.3 until all weights are computed.

S2.2.6 - Remove the redundant or inefficient knots - i.e. knots associated to a criterion value close to the reference one $J_{\text {ref }}^{\prime}$ (i.e. $\left(J_{k n o t}^{\prime}-J_{\text {ref }}^{\prime}\right) / J_{\text {ref }}^{\prime}<0.001$ ).

S2.2.7 - Go back step S2.2.2 until it is not possible to delete a knot.

As complementary computational considerations, if adjacent knots (along the $x$ axis or the $y$-axis) have a criterion value close to the reference one, only the closer one is removed. In fact, when a knot is removed from the knot distribution, the weight of the neighboring knots change. So, after each knot removal, the weight of each knot of the new distribution must be computed again. Moreover, each minimization of the criterion $J^{\prime}$ is achieved for a given limited number of iterations (once again, here, one just looks for a weight of each knot). 
The proposed knot insertion and deletion schemes have been constructed to determine an effective knot distribution for representing the unknown object. This approach enables us to insert knots essentially within the area of the object and hence introduces some "geometrical information" about the unknown. Although not necessarily optimal, this method is quite well adapted to the inverse scattering problem and in what follows, the results obtained with simulated data and real data show the efficiency and accuracy of the proposed method.

\section{Numerical results}

In this section, in order to show the potential of the proposed adaptive B-spline method and the specific knot insertion and deletion procedures, results obtained with simulated data are presented.

\subsection{Adaptive reconstruction}

The configuration under study [11] consists of homogeneous dielectric cylinders with a $4 \lambda / 5$ sided square cross-section, separated by a distance of approximatively $\lambda / 2$. The contrast of these cylinders is $\chi=1$. . The square search domain, of side $d=3 \lambda$, is discretized into $29 \times 29$ cells. $L=29$ electromagnetic excitations and $M=29$ receivers are considered. The simulated object (see Figure 4a) and the retrieval ones are presented through amplitude and/or gray level maps with wavelength-normalized scale axis.

To initialize the algorithm, a knot collection comprised of: $k_{x}=[1,7,14,21,29]$ and $k_{y}=[1,7,14,21,29]$ is considered. This initial knot distribution does not take into account any a priori information on the objects locations and allows us to obtain a homogeneous coarse representation of the domain (see Figure 6a). Figure 4 shows the reconstructions obtained after each non-linear inversion, for a threshold fixed at $T_{h}=0.80$. Figure $4 \mathrm{~b}$ shows the solution obtained after the first non-linear inversion (step S1.3), which required 5 iterations and corresponded to the first knot distribution (see Figure 6a). Figures 4c,d,e,f show the results at the end of each stage S2.4, where 10 iterations of the non-linear conjugate-gradient (CG) method were used in all cases.

The final knot distribution consists of: $k_{x}=[1,4,4.75,6.43,8.75,9.62,10.50$, $14.00,18.37,19.25,20.12,21.75,22.50,23,25,29]$ and $k_{y}=[1,7,10.50,11.37,12.25$, 14.87, 15.75, 16.62, 17.50, 21, 29] (see Figure 6b). Thus, the final retrieved object is represented by 176 control points requiring the determination of only 27 knots in the B-spline approach instead of 841 pixels for the pixel-based approach.

Figure 5 shows the simulated object, the reconstructions obtained using 45 iterations of the conjugate-gradient method (presented in section 3) where the unknowns are the full set of 841 pixel values, and the results of the adaptive B-spline approach. One can notice a slight enhancement of the final solution, particularly for the shape of the object, using the adaptive approach which requires only $27 / 841 \approx 0.032$ degrees of freedom relative to a pixel-based reconstruction. 


\subsection{Noise effect}

In this subsection, in order to show the influence of the noise on the reconstructions, the previous configuration is considered with noise added to the scattered field as follows:

$$
\mathbf{e}_{b}^{d}=\mathbf{e}^{d}(1+r \exp (i \phi))
$$

where $r$ is the percentage of added noise and $\phi$ is a vector of random deviates in the range $[0,2 \pi]$.

To compare the reconstructions, depending on the noise level, the Peak Signal to Noise Ratio $(P S N R)$, commonly used in image processing, is considered. It is defined for a gray level $B$ bits by:

$$
P S N R=10 \log \left(\frac{\left(2^{B}-1\right)^{2}}{d}\right),
$$

where $d$ is the mean quadratic error given for a $\left[N_{x} \times N_{y}\right]$ image by:

$$
d=\frac{1}{N_{x} N_{y}} \sum_{i=0}^{N_{x}-1} \sum_{j=0}^{N_{y}-1}\left(I_{0}(i, j)-I_{1}(i, j)\right)^{2},
$$

where $I_{0}$ is the initial image (simulated object) and $I_{1}$ is the reconstructed image.

Figure 7 shows the evolution of the $P S N R$ for the adaptive approach (dashed line) and the pixel-based approach (black line) for various noise levels. From these results one can observe that the reconstructions obtained with the adaptive approach are better than the pixel-based reconstructions. Hence by controlling the complexity of the inversion process using our B-spline technique one obtains a degree of robustness that does not require the use of an explicit regularization functional.

\subsection{Effect of Threshold, $T_{h}$}

Here some results are presented to show the effect of the one key free parameter, $T_{h}$, on the reconstruction process.

The target is comprised of two dielectric cylinders with circular cross-section of radius $r=15 \mathrm{~mm}$. One is characterized by $\chi=3$ and the other one by $\chi=1.8$. The search domain is a rectangular area $9.6 \times 14.4 \mathrm{~cm}^{2}$ sized and it is discretized into $24 \times 36$ cells. The imaging system consists in 36 sources and 72 receivers and the operation frequency is $f=6 \mathrm{GHz}$.

The initial knot distribution consists of $k_{x}=[1,5,10,15,20,24]$ and $k_{y}=[1,7$, 14, 21, 29, 36]. Figure 8 shows the simulated object and the obtained reconstructions using the pixel-based approach and the adaptive scheme for various threshold values. The adaptive process causes new knots to be introduced only in the area of the higher permittivity object which leads to some degradation in the reconstruction of the smaller contrast object relative to the pixel-based approach. On these simulated data, the reconstructions for two successive thresholds are close but it was noticed that it is not always true particulary for real data. Moreover, some artifacts appear in the regions where there is no object. One can think that they are due to our global representation 
of the search domain - the obtained knot distribution is optimal for the representation of the objects but this distribution leads to a non-efficient representation of the regions where there is no object.

Finally, the number of elements to be estimated in the adaptive approach is about 120 to 132 , depending on the threshold level, to be compared with the 864 elements for the pixel-based method.

\section{Application to real data}

The experimental data used in this paper were carried out at Institut Fresnel (Marseille - France) and have been used to test a wide range of inversion schemes. One can particulary refer to the special section: "Testing inversion algorithms against experimental data," of the journal Inverse Problems (December 2001). Hence, the proposed adaptive scheme and the obtained results can be compared with these different approaches.

The considered experimental setup (see Figure 9), is described in [4]. A dielectric or metallic homogeneous object is irradiated by $L=36$ different locations evenly distributed around the object. The TM polarized incident fields, $e_{l}^{i n c}(l=1, \ldots, L)$, are modelled in the investigating domain by a linearly polarized isotropic cylindrical wave as defined in (1). The scattered field for each irradiation $e_{l}^{d}$ is measured for $M=72$ different locations evenly distributed around the object. However, due to physical limitations, there is a blind zone of $60^{\circ}$, from each part of the emitter, such that the scattered field is measured for 49 out of the 72 receiver angles.

\subsection{Two dielectric objects}

A dielectric target made of two identical cylinders with circular cross-section of radius $1.5 \mathrm{~cm}$ is considered here. The relative permittivity of this target was estimated to $\varepsilon_{r}=3 \pm 0.3$ [4]. The data associated to the so-called twodielTM_8f.exp file are considered for two frequencies corresponding to $4 \mathrm{GHz}$ and $7 \mathrm{GHz}$. In both cases, a rectangle search domain of $8 \mathrm{~cm}$ (along the $x$-axis) $\times 16 \mathrm{~cm}$ (along the $y$-axis), discretized into $20 \times 40$ cells and centered at $(x=0 \mathrm{~cm}, y=0 \mathrm{~cm})$ is considered.

Figure 10 shows the reconstructions obtained after 65 iterations of the conjugate gradient algorithm and the reconstructions obtained using the adaptive B-spline approach. For this method, 5 iterations of the nonlinear CG method are used in the first inversion step S1.3, and in the 5 iterations of step $\mathbf{S 2 . 4} 12$ iterations are employed. For both frequencies, the initial knot distribution consists of: $k_{x}=[1,5,10,15,20]$ and $k_{y}=[1,10,20,30,40]$ and the threshold is fixed at $T_{h}=0.60$.

At $4 \mathrm{GHz}$, the maximum value of the reconstructed permittivity is $\varepsilon_{r}=4.53$ for the conjugate-gradient method and $\varepsilon_{r}=3.48$ for the adaptive approach - to be compared with $\varepsilon_{r}=3 \pm 0.3$. The final knot distribution is: $k_{x}=[1,7.50,9.37,11.25,11.87,12.50$, $13.12,13.75,15,20]$ and $k_{y}=[1,7.75,10,10.62,11.25,15,20,25,29.37,30,31.25,35$, 
40]. The final estimated object is represented by 130 control points through 23 knots in the B-spline approach instead of 800 pixels. For this frequency, the two reconstructions are similar but the estimated permittivity is better with the adaptive approach.

At $7 \mathrm{GHz}$, the maximum value of the reconstructed permittivity is $\varepsilon_{r}=4.23$ for the conjugate-gradient method and $\varepsilon_{r}=4.10$ for the adaptive approach. The final knot distribution is: $k_{x}=[1,5,7.50,8.12,8.75,9.37,10,10.62,11.25,11.87,12.50,13.12$, $13.75,20]$ and $k_{y}=[1,5.50,8.31,8.87,9.43,10,10.62,11.25,11.87,15,25,26.25,27.50$, $28.12,28.75,29.37,30,30.62,31.25,35,40]$. The final estimated object is represented by 294 control points through 35 knots in the B-spline approach instead of 800 pixels. The enhancement of the reconstruction using the adaptive approach is clearly visible on these results.

One can notice that the center of the reconstructed cylinders, for both frequencies, is slightly shifted. This shift, observed in all previous results (see the special section of Inverse Problems), is within the experimental margin.

\subsection{Metallic rectangular object}

The metallic target is a centered filled cylinder with a rectangular cross-section of $1.27 \times 2.54 \mathrm{~cm}^{2}$. The considered experimental data correspond to the so-called rectTM_cent.exp file for $8 \mathrm{GHz}$ and $16 \mathrm{GHz}$. For the search domain, a $2.1 \mathrm{~cm} \times 3.2$ $\mathrm{cm}$ area, discretized into $20 \times 30$ cells and centered at $(x=-0.5 \mathrm{~cm}, y=-0.75 \mathrm{~cm})$ is considered. The results are presented as gray level maps of the normalized non-negative imaginary part of the contrast functions, since only the shape is of interest for such impenetrable object.

Figure 11 shows the obtained reconstructions after 45 iterations using the conjugate gradient algorithm and the adaptive scheme. For both frequencies, the initial knot distribution is: $k_{x}=[1,5,10,15,20]$ and $k_{y}=[1,10,20,30]$ and the threshold is fixed at $T_{h}=0.8$.

At $8 \mathrm{GHz}$, the final knot distribution is $k_{x}=[1,5,6.25,7.50,8.12,8.75,9.37,10$, $11.25,13.75,15,20]$ and $k_{y}=[1,5.50,10,11.25,12.50,13.75,14.37,15,16.25,16.87$, $17.50,18.75,20,30]$. The final estimated object is represented by 168 control points through 26 knots in the B-spline approach instead of 600 pixels.

At $16 \mathrm{GHz}$, the final knot distribution is $k_{x}=[1,5,7.50,10,11.25,12.18,13.12$, $13.75,15,20]$ and $k_{y}=[1,5.50,10,10.62,11.25,12.18,13.12,14.37,15,15.62,16.25$, $16.87,17.50,18.75,20,25,30]$. The final estimated object is represented by 170 control points through 27 knots in the B-spline approach instead of 600 pixels.

As the object to be estimated is impenetrable, only its edges are expected to be retrieved like for the results at $16 \mathrm{GHz}$. So, at $8 \mathrm{GHz}$ it seems reasonable to consider that the results correspond to an equivalent object. One can also notice that the resolution is not very good in some parts of the object for the reconstructions obtained with the adaptive approach. One can also think that it is due to our global representation. 


\section{3. "U-shaped" metallic object}

In this part, a "U-shaped" metallic cylinder defined within a $8 \times 5 \mathrm{~cm}^{2}$ rectangle is considered. The corresponding experimental data are the so-called uTM_shaped.exp file for $16 \mathrm{GHz}$. The considered centered search domain is a $15 \mathrm{~cm} \times 12 \mathrm{~cm}$ area discretized into $50 \times 40$ cells. Like for the previous target, the results are presented as gray level maps of the normalized non-negative imaginary part of the contrast.

Figure 12 shows the discretized search domain, the reconstructions obtained using the conjugate gradient and using the adaptive approach for a threshold fixed at $T_{h}=0.70$. The initial knot distribution consists of $k_{x}=[1,10,20,30,40,50]$ and $k_{y}=[1,10,20,30,40]$. The final knot distribution, is $k_{x}=[1,10,10.75,11.50,12,23.50$, $36.40,36.72,38,38.92,40]$ and $k_{y}=[1,10,11.42,12,14.75,17.52,18,22.75,25.52,26.50$, $27.68,28,28.5,29.25,29.75,30,30.50,31.25,40,50]$. So the search domain is described by 210 control point through 31 knots instead of 2000 pixels.

Here, one can notice the very good reconstruction obtained using the adaptive approach and the very small number of element to be estimated with the adaptive approach in comparison with the pixel-based method. Moreover, some artifacts can be seen inside the "U" they appear to be due to stationary wave created inside the cavity.

\section{Conclusion}

In this paper, an adaptive spline-based approach for solving inverse scattering problems has been presented. This inversion method has been proposed in order to reduce the complexity of this non-linear, and ill-posed inverse problem. In addition our experiments indicate that the reconstructions can be improved in comparison with a simple pixelbased representation of the object. The implementation of this method allows us to solve the inverse problem by determining the knot distribution which approximates the object to be estimated. In order to estimate the knot distribution a specific knot insertion process, based on curvature information, and a deletion process, based on data fitting information, have been proposed. We demonstrate its utility both in the processing of simulated data and real data. Thus the feasibility and utility of the underlying approach of spline-based adaptive processing for inverse problem is validated. Moreover, this approach has been also applied for the detection and characterization of buried objects in an half-space using simulated data [2].

An area of current interest is the development of more rigorous methods for performing knot refinement in order to improve the results and the computational time. Indeed, the small number of elements to be estimated leads to decrease the computational time in the minimization procedure however; the actual deletion scheme can be time-consuming. In an other way, a more localized description of the image is under consideration in order to avoid some artifacts or the loss of resolution. In future works, the use of Non-Uniform Rational B-Splines (NURBS) could be an interesting area of studies for the enhancement of the approach. 


\section{Acknowledgment}

The authors want to acknowledge D. Lesselier from the 'Laboratoire des Signaux et Systèmes' (L2S - CNRS/SUPELEC/UPS) for fruitful discussions and useful readings of the manuscript.

This work was supported in part by CenSSIS, the Center for Subsurface Sensing and Imaging Systems, under the Engineering Research Centers Program of the National Science Foundation (award number EEC-9986821) as well as a NSF Award \#0208548 and in part by SATIE supported by the French ministry of research and the CNRS (Centre National de la Recherche Scientifique).

\section{References}

[1] R.H. Bartels, J.C. Beatty and B.A. Barsky, An Introduction to Splines for Use in Computer Graphics and Geometric Modeling. Morgan Kaufmann, 1987.

[2] A. Baussard, E.L. Miller, Detection and characterization of buried objects using an adaptive Bspline scheme, 2003 IEEE Conference on Geoscience and Remote Sensing (IGARSS), Toulouse, vol. 6, pp. 3833-3835, July 2003.

[3] R.C. Beach, An Introduction to the Curves and Surfaces of Computer-Aided Design. Van Nostrand Reinhold, 1991.

[4] K. Belkebir and M. Saillard, "Special section: Testing inversion algorithms against experimental data," Inverse Problems, vol. 17, pp. 1565-1571, 2001.

[5] A. Davies and P. Samuels, An Introduction to Computational Geometry for Curves and Surfaces. Oxford University Press (New York), 1996.

[6] P. Dierckx, Curve and Surface Fitting with Splines. Oxford University Press (New York), 1993.

[7] C. Dourthe, C. Pichot, J.Y. Dauvignac, L. Blanc-Féraud and M. Barlaud, "Regularized biconjugate gradient algorithm for tomographic reconstruction of buried objects," Special issue on problems on random scattering and electromagnetic wave sensing, IEICE Trans. on Electronics, vol. E83-C, no. 12, pp. 1858-1863, 2000.

[8] N. Joachimowicz, C. Pichot and J.P. Hugonin, "Inverse scattering: An iterative numerical method for electromagnetic imaging," IEEE Trans. Antennas Propagat., vol. 39, pp. 1742-1751, 1991.

[9] G. Farin, Curves and Surfaces for Computer Aided Geometric Design. A Practical Guide. fourth edition, Academic Press, 1997.

[10] F.R. Harrington, Field Computation by Moment Methods. Macmillan (New York), 1968.

[11] R.E. Kleinman and P.M. van den Berg, "A modified gradient method for two dimensional problems in tomography," J Comput Appl Math, vol. 42, pp. 17-35, 1992.

[12] P. Lobel, R.E. Kleinman, C. Pichot, L. Blanc-Féraud and M. Barlaud, "Conjugate gradient method for solving inverse scattering with experimental data," IEEE Antennas Propag. Mag., vol. 38(3), pp. 48-51, 1996.

[13] D. Macías, G. Olague, E.R. Mèndez, Hybrid Evolution Strategy-Downhill Simplex Algorithm for Inverse Light Scattering Problems, Application of Evolutionary computing - 5th European Workshop on Evolutionary Computation in Image Analysis and Signal Processing, S.Cagnoni et al., LNCS 2611, pp. 399-409, Springer-Verlag Berlin Heidelberg, 2003.

[14] E.L. Miller, M. Kilmer, C. Rappaport, "A new shape-based method for object localization and characterization from scattered field data," IEEE Trans. Geosci. Remote Sensing, vol. 38, no. 4, pp. 1682-1696, 2000.

[15] E.L. Miller, I. Yavuz, L. Nicolaides and A. Mandelis, "An adaptive multiscale inverse scattering approach to photothermal depth profilometry," Circuits, Systems, and Signal Processing, special issue on advanced signal/image restoration, vol. 19, no. 4, pp. 339-363, August 2000. 
[16] W.H. Press, B.P. Flannery, S.A. Teukolski and W.T. Vetterling, Numerical Recipes. The Art of Scientific Computation. Cambridge University Press (Cambridge), 1986.

[17] P.M. van den Berg and R.E. Kleinman, "A contrast source inversion method," Inverse Problems, vol. 13, pp. 1607-1620, 1997. 


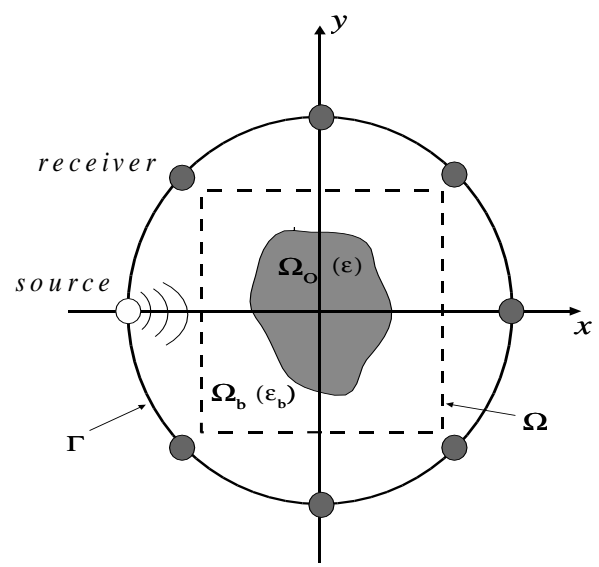

Figure 1. Geometry of the problem.
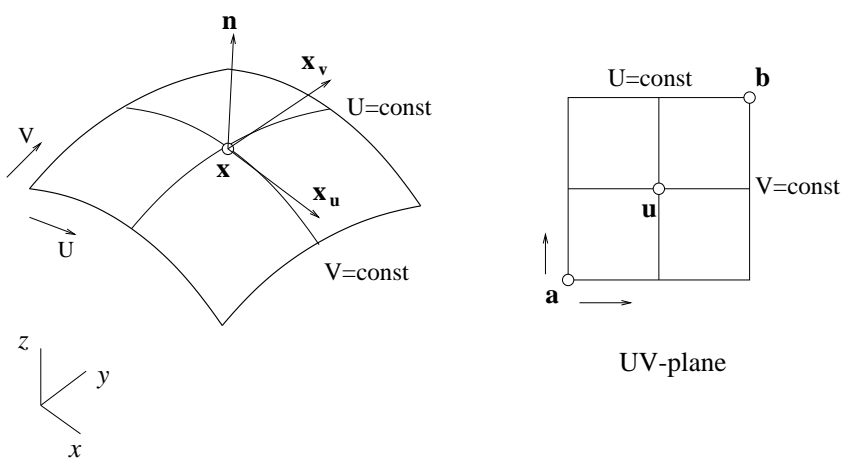

Figure 2. A parametric surface.

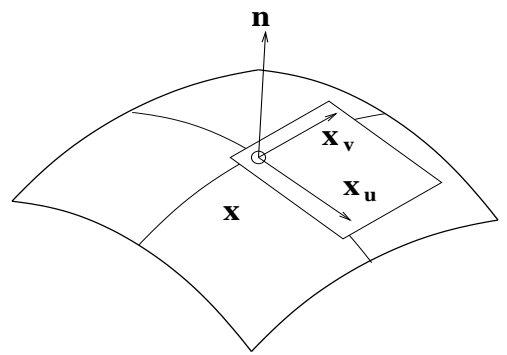

Figure 3. Local frame and tangent plane. 


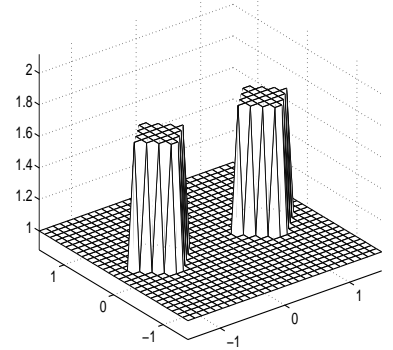

(a)

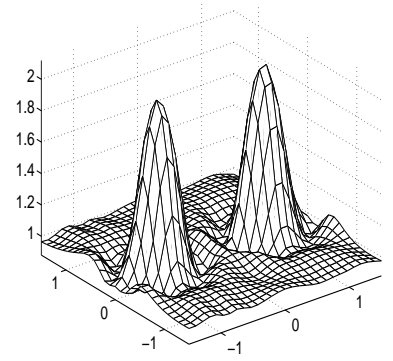

(d)

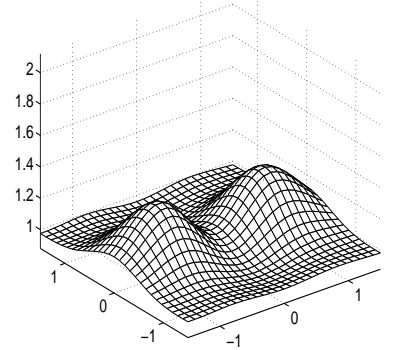

(b)

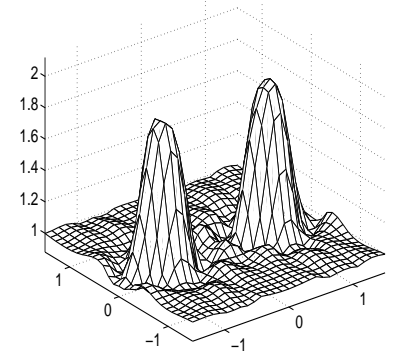

(e)

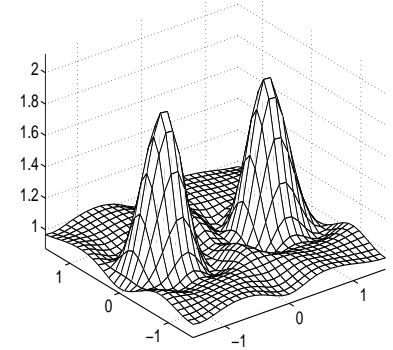

(c)

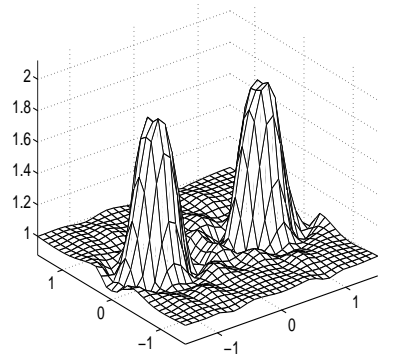

(f)

Figure 4. (a) Real part of the simulated object. (b) Initial reconstruction obtained at the end of stage S1.3. (c,d,e,f) Successive reconstructions obtained at the end of each stage $\mathbf{S 2 . 4}$.

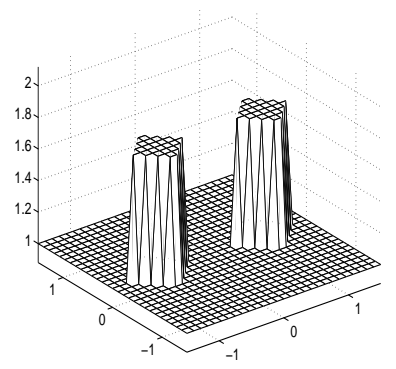

(a)

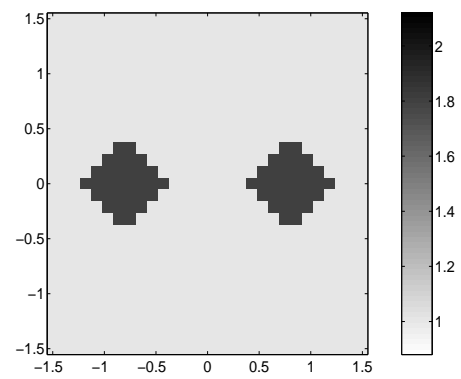

(b)

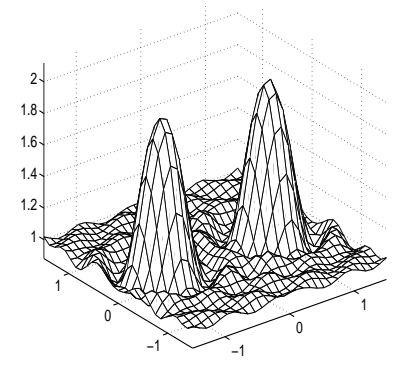

(c)

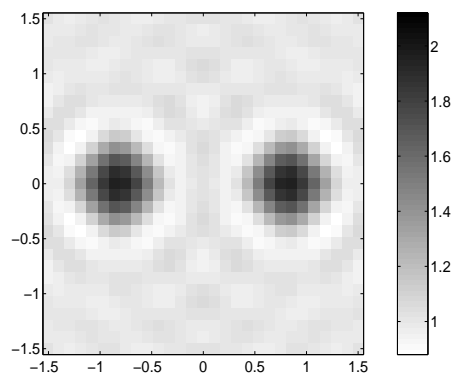

(d)

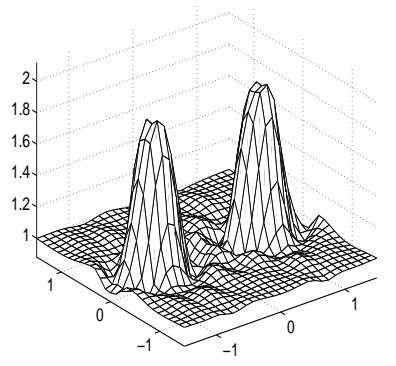

(e)

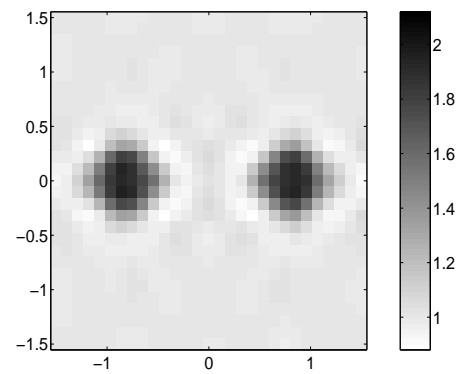

(f)

Figure 5. (a) Mesh and (b) gray level map of the simulated object. (c,d) Reconstruction obtained using the conjugate gradient algorithm. (e,f) Reconstruction obtained using the adaptive approach (same as in figure $4 \mathrm{f}$ ). 


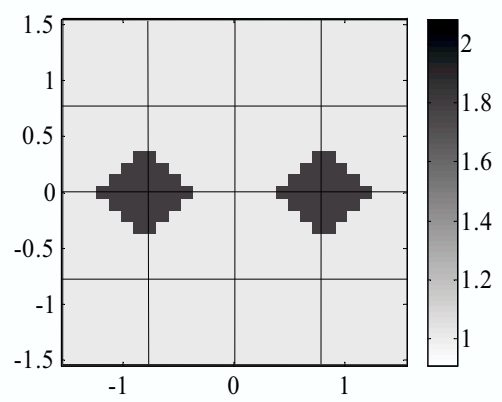

(a)

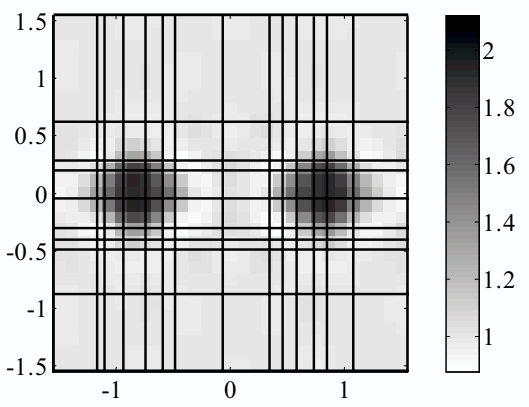

(b)

Figure 6. (a) Initial knot distribution display over the simulated object (Figure 5b). (b) Final knot distribution display over the final reconstruction (cf figure 5f).

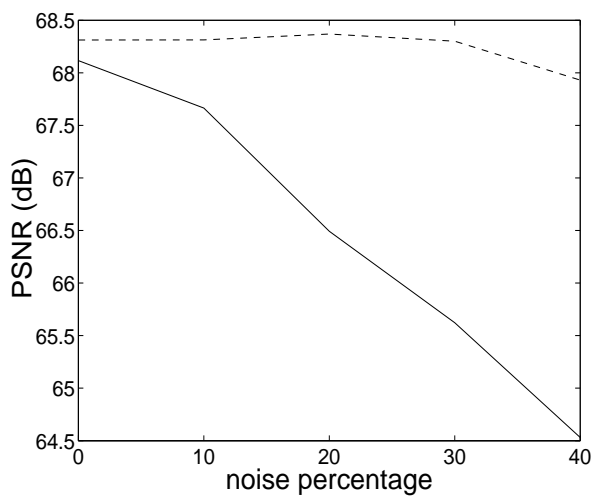

Figure 7. PSNR evolution for (-) the conjugate-gradient method and for (- -) the adaptive approach depending on the added percentage of noise. 


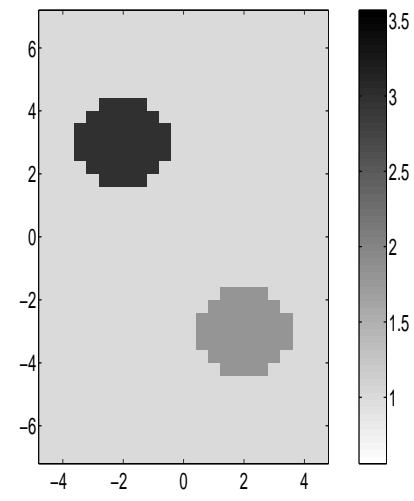

(a)

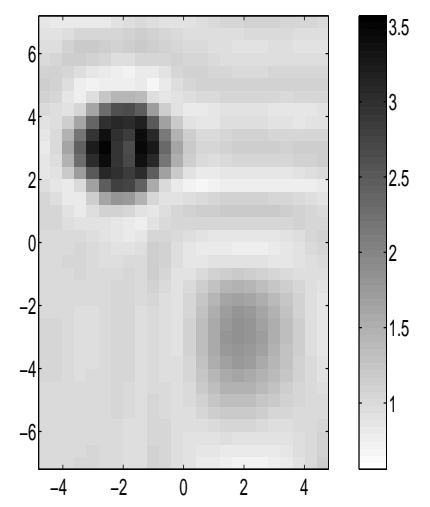

(d)

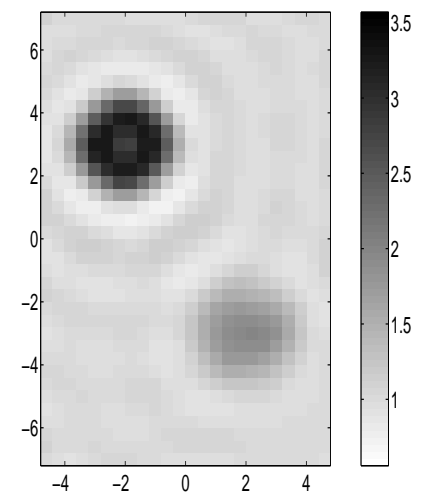

(b)

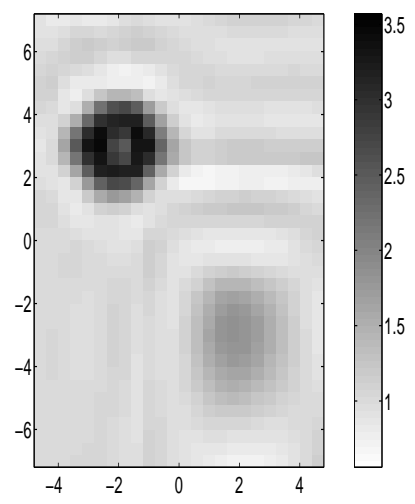

(e)

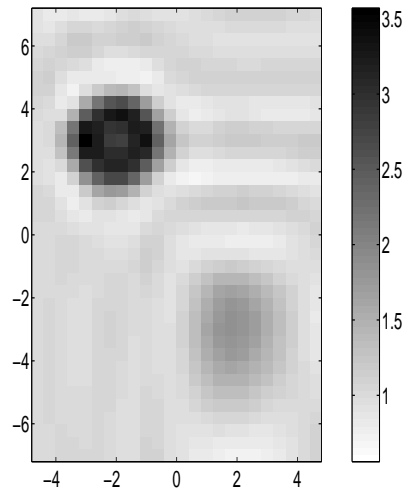

(c)

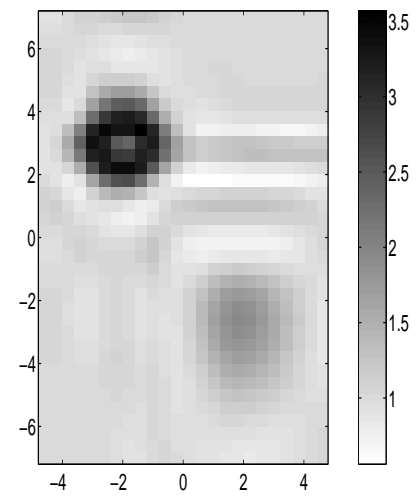

(f)

Figure 8. (a) Simulated object. Reconstructions obtained using (b) the conjugate gradient approach and the adaptive approach for (c) $T_{h}=0.6 \%$, (d) $T_{h}=0.7 \%$, (e) $T_{h}=0.8 \%$ and (f) $T_{h}=0.9 \%$. 


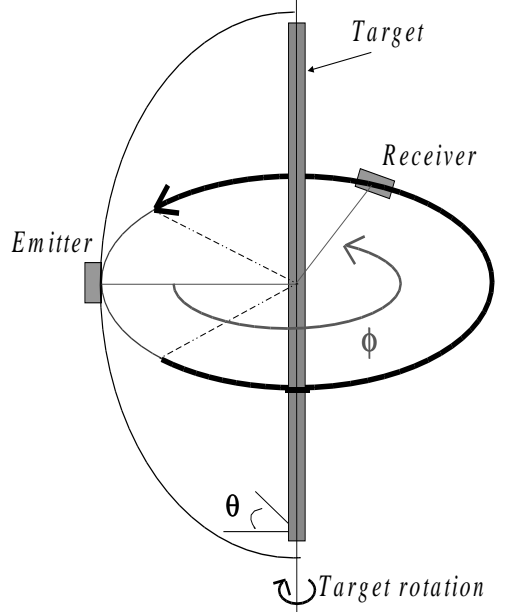

Figure 9. Experimental setup geometry used for validating the inverse algorithm. $\phi$ denotes the angle of receiving antenna while $\theta$ represents the angle of the emitting antenna. 


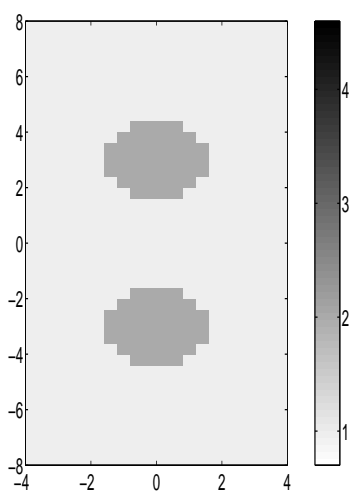

(a)

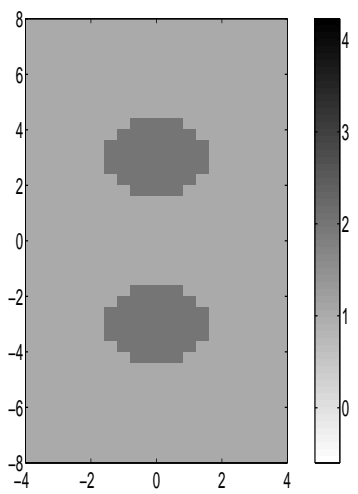

$(d)$

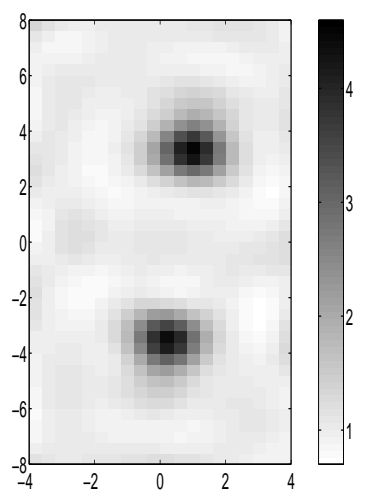

(b)

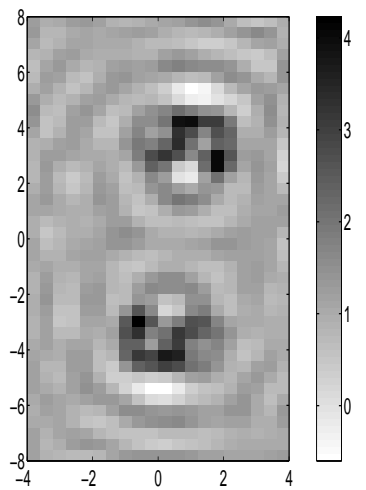

(e)

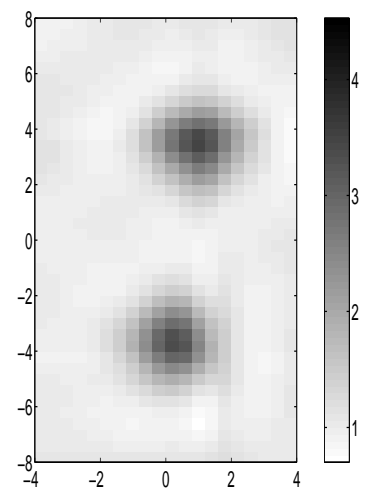

(c)

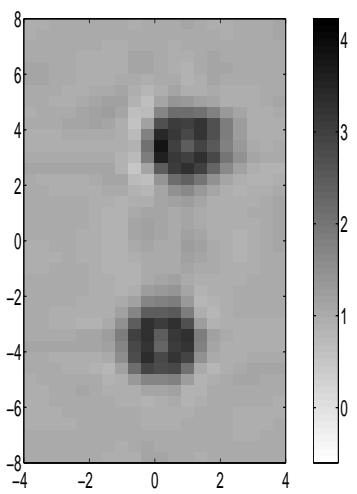

(f)

Figure 10. Gray scale images of the reconstructed dielectric target made of two cylinders at (a,b,c) $4 \mathrm{GHz}$ and (d,e,f) $7 \mathrm{GHz}$, within a $8 \mathrm{~cm} \times 16 \mathrm{~cm}$ centered search domain discretized into $20 \times 40$ cells. (a,d) Simulated object, (b,e) reconstructions obtained with the conjugate-gradient method, and (c,f) reached solutions by the adaptive approach. 


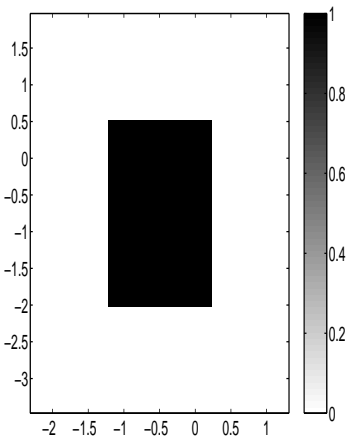

(a)

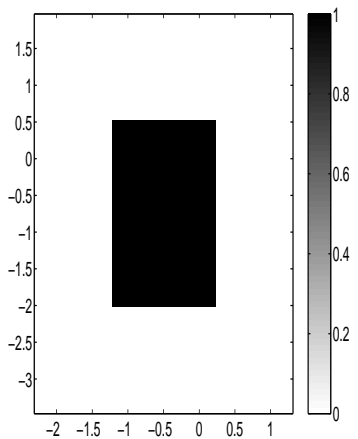

(d)

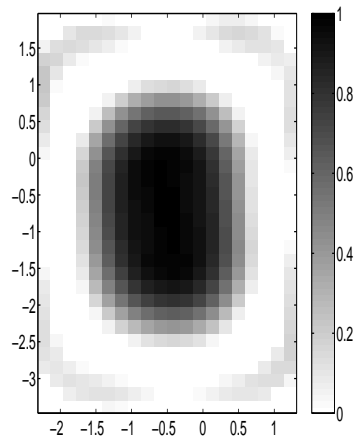

(b)

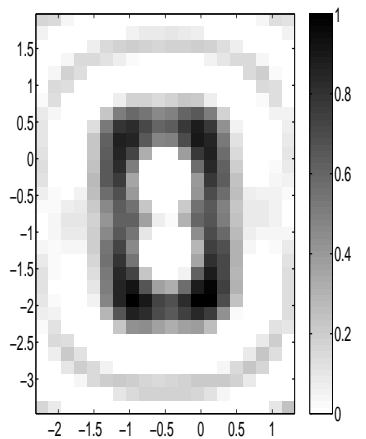

(e)

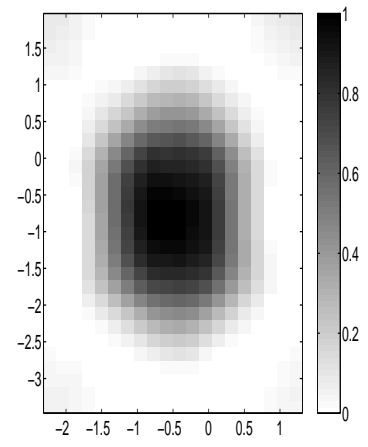

(c)

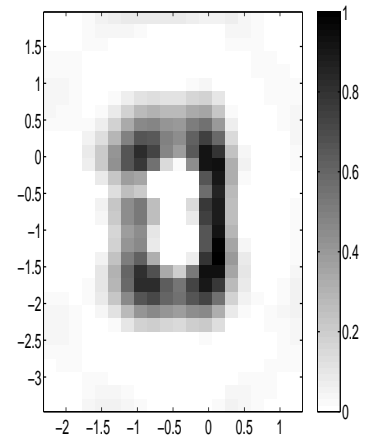

(f)

Figure 11. Reconstruction of the metallic target at (a,b,c) $8 \mathrm{GHz}$ and (d,e,f) $16 \mathrm{GHz}$, within a $2.1 \mathrm{~cm} \times 3.2 \mathrm{~cm}$ centered search domain discretized into $20 \times 30$ cells. (a,d) Simulated target, (b,e) reconstructed object with the conjugate-gradient method, and $(\mathrm{c}, \mathrm{f})$ reconstructions obtained using the adaptive approach.

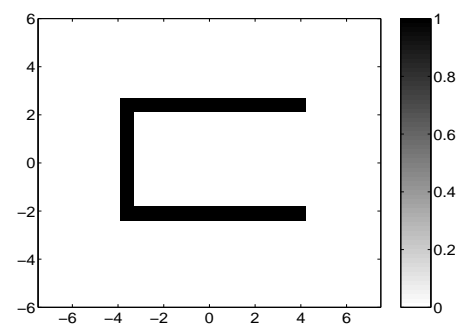

(a)

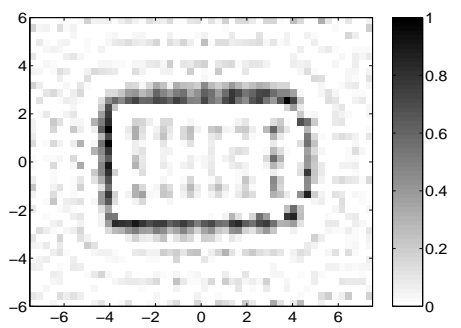

(b)

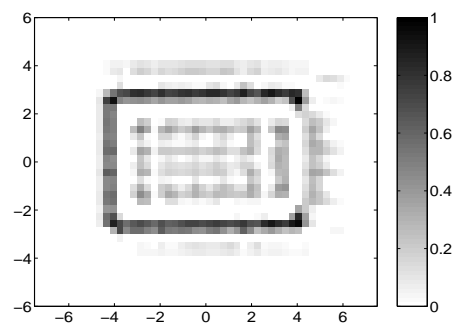

(c)

Figure 12. Reconstruction of the "U-shaped" metallic target at $16 \mathrm{GHz}$, within a 15 $\mathrm{cm} \times 12 \mathrm{~cm}$ centered search domain discretized into $50 \times 40$ cells. (a) simulated target (b) reconstructed target with the conjugate-gradient algorithm, and (c) reconstruction obtained using the adaptive approach. 